\title{
Safety and Efficacy of Transbrachial Access for Coronary Procedures: Case Report and Review of Literature
}

\author{
Mehssani Z*, Koné GH, Bounssir A, Fellat R PR, Srairi J PR and Fellat N PR \\ Department of Cardiology, A/National Cardiovascular League, Ibn Sina University Hospital Center, Mohammed V University, Rabat.
}

\begin{abstract}
*Corresponding author: Mehssani Z, Department of Cardiology, A/National Cardiovascular League, Ibn Sina University Hospital Center, Mohammed V University, Rabat.
\end{abstract}

Received Date: May 18, 2019

Published Date: June 22, 2020

\section{Introduction}

Trans radial approach (TRA) has gained popularity over the trans femoral access (TFA) and has become the default option for coronary procedures around the world, as it has less bleeding access site complications and it is associated with a lower degree of adverse clinical events rate. However, in some situations, the trans radial arterial access could be unsuccessful. We present a case of a 66 years old man with history of aortobifemoral bypass surgery admitted in our department for an ACS and neither TFA nor TRA access were possible.

\section{Case Report}

We describe a case of a 66 years old male, with an aortoiliac occlusive disease who was admitted to the vascular surgery unit for aortobifemoral bypass surgery. He had a significant medical history of hypertension, diabetes and an extensive smoking. Pre-operative CAD screening including stress test was normal. The day following the vascular surgery, the patient suffered from severe angina with EKG abnormalities and troponin elevation. He was diagnosed with a non-ST-segment elevation myocardial infarction (NSTEMI). He was forthwith referred to our department for an angiogram to assess for coronary artery disease that may require revascularisation therapy. In the Cath Lab, the radial artery pulse was non palpable due to the thick arterial wall and a low flow state, puncture and cannulation on both radial arterial sides were challenging. In addition, the transfemoral access was not feasible knowing that the patient has just undergone an artobifemoral graft surgery one day ago (Figures 1\&2).

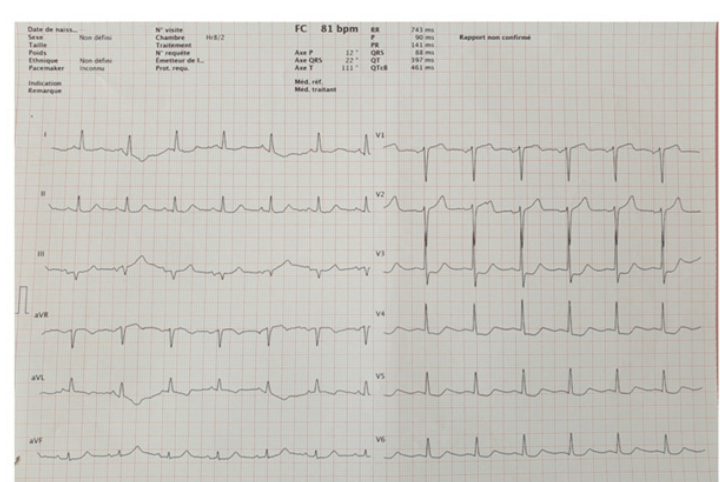

Figure 1: ECG showing sinus rhythm with apical and lateral ST segment depression.

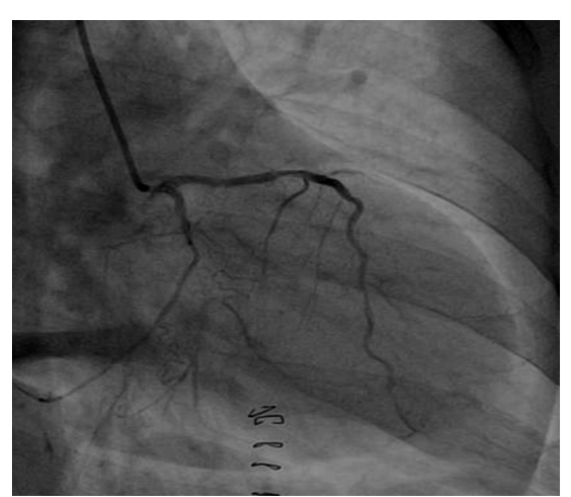

Figure 2: Left coronary angiography RAO caudal : left main stenosis, severe circumflex stenosis and mid LAD stenosis. 
To overcome this technical problem, the right brachial artery as an alternative vascular access has been attempted. $1 \mathrm{ml}$ of lidocaine was injected into the subcutaneous tissues overlying the right brachial artery (Figure 3).

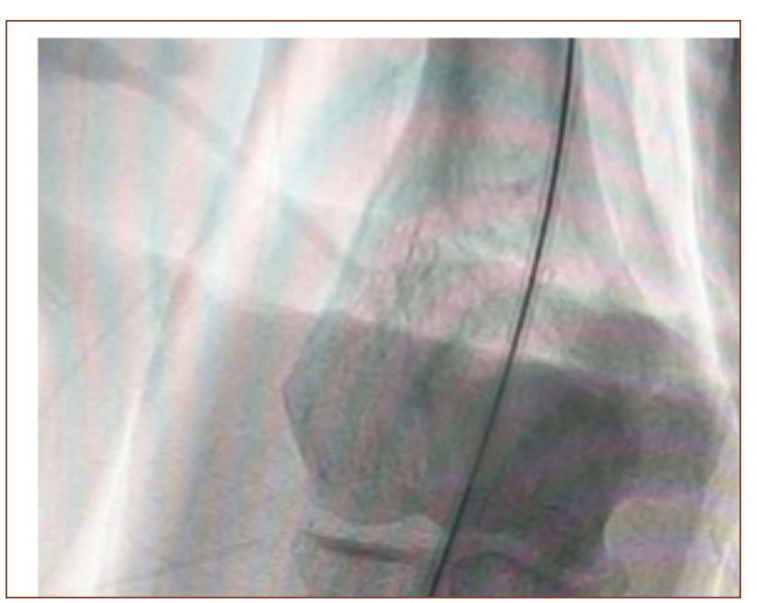

Figure 3: Fluoroscopy of the $6 \mathrm{~F}$ seath and guidewire into the right brachial artery.

A Terumo needle dedicated to radial access was used and a $6 \mathrm{~F}$ sheath (Terumo interventional system) was pushed easily over an hydrophilic wire and inserted for diagnostic purpose and ad-hoc intervention depending on needs, the intra-arterial nitroglycerine usually used in TRA access to prevent spasm was not administered, and 3000 UI of unfractionned heparin was injected into the sheath sidearm. Judkin's left and right catheters were successfully advanced into the ascending aorta enabling left main and right coronary engagement and angiography without any difficulties in catheter manipulation during the procedure.

Severe CAD was diagnosed with left main disease, mid LAD stenosis, severe obtuse marginal stenosis and a severe calcified lesion of the RCA ostium. CABG was proposed in order to perform complete revascularisation. After the procedure, the sheath was removed and hemostasis was done by digital compression for 15 min. The puncture site was bandaged and a hard support has been placed under the arm to limit movements of the elbow, with very close monitoring during the first 6 hours.

\section{Discussion}

Trans radial arterial approach (TRA) coronary angiogram reduces vascular complications compared with trans femoral approach (TFA). However, the mechanism and predictors of TRA failure access have not been well characterized (Congenital radial artery atrophy/hypoplasia, tortuous configurations, radioulnar loop and abnormal origin of the radial artery; damage to the radial artery and loss of pulse). Many interventional cardiologists considered that switching to the TBA is outdated and risky access. It has been demonstrated significantly higher rate of vascular and neurological complications than other access in previous studies [1]. Nevertheless, with additional care by using smaller sized catheters, an adequate anticoagulation regimen proper methods for achievement of haemostasis as well as a close and careful observation afterward, Newcomers are trying to overcome this mindset and make the TBA approach possible when the TRA fails [2].

Sabbah et al [3] studied 4955 cases undergoing coronary revascularization. Respectively, $22 \%, 57 \%$ and $21 \%$ cases were divided into TBA, TRA, and TFA groups. Results showed that forearm artery access in TBA and TRA groups was associated with higher procedural success compared with TFA group . Compared with TRA group, TFA group had higher rate of MACE and inhospital cardiac death, and also higher incidence of major access site hematoma. TBA and TFA groups had higher rate of access site pseudoaneurysm. Gan et al [1] studied 5110 cases undergoing coronary procedures. Of these, 180 and 121 cases were enrolled to TBA and TFA groups, respectively.

Results showed TBA group had a slighter lower incidence of major complications compared with TFA group without significant difference even in minor complications. There was no incidence of brachial artery thrombosis and no puncture-related neurological dysfunction in TBA group.

\section{Conclusion}

Our case alongside cited studies above provides evidence that the brachial approach has to be considered as an efficient alternative to the femoral approach for coronary angiogram and interventional procedures when radial access is unsuccessful.

\section{Acknowledgement}

None.

\section{Conflict of Interest}

No conflict of interest.

\section{References}

1. Hwa WG, Hon KY, Chiung JW (2010) Brachial approach for coronary angiography and intervention : Totally obsolete, or a feasible alternative 
when radial access is not possible? Ann Acad Med Singapore 39(5): 368373.

2. Lam UP, Lopes Lao EP, Lam KC, Evora M, Wu NQ (2019) Trans-brachial artery access for coronary artery procedures is feasible and safe: data from a single-center in Macau. Chin Med J 132(12): 1478-1481.
3. Sabbah M, Kadota K, Fuku Y, Goto T, Habara S, et al. (2013) Comparative effectiveness of the different arterial approaches transbrachial, transradial and transfemoral in percutaneous coronary interventions: real-world experiences. J Am Coll Cardiol 62: B89. 